\title{
A Vortex Lattice Method for the Hydrodynamic Solution of Lifting Bodies Traveling Close and Across a Free Surface
}

\author{
RAFFAELE SOLARI ${ }^{1}$, PATRIZIA BAGNERINI ${ }^{2}$, GIULIANO VERNENGO ${ }^{1}$ \\ ${ }^{1}$ Department of Electric, Electronic, Telecommunication Engineering and Naval Architecture (DITEN) \\ Polytechnic School of the University of Genoa, \\ Via Montallegro 1, 16145, GE, ITALY \\ ${ }^{2}$ Department of Mechanical, Energy, Management and Transportation Engineering (DIME) \\ Polytechnic School of the University of Genoa, \\ Via all'Opera Pia 15, 16145, GE, ITALY
}

\begin{abstract}
The hydrodynamics performance of submerged and surface-piercing lifting bodies is analyzed by a potential flow model based on a Vortex Lattice Method (VLM). Such a numerical scheme, widely applied in aerodynamics, is particularly suitable to model the lifting effects thanks to the vortex distribution used to discretize the boundaries of the lifting bodies. The method has been developed with specific boundary conditions to account for the development of steady free surface wave patterns. Both submerged bodies, such as flat plates and hydrofoils, as well as planing hulls can be studied. The method is validated by comparison against available experimental data and other Computational Fluid Dynamic (CFD) results from Reynolds Averaged Navier Stokes (RANS) approaches. In all the analyzed cases, namely 2D and 3D flat plates, a NACA hydrofoil, planning flat plates and prismatic planing hulls, results have been found to be consistent with those taken as reference. The obtained hydrodynamic predictionsare discussed highlighting the advantages and the possible improvements of the developed approach.
\end{abstract}

Key-Words: Vortex Lattice Method (VLM); Boundary Element Method (BEM); Free Surface; Hydrofoils; Planing Surfaces.

Received: April 21, 2021. Revised: January 15, 2022. Accepted: January 27, 2022. Published: March 8, 2022.

\section{Introduction}

Design high performance floating and submerged vessels has always been a great challenge in hydrodynamics. This is mainly related to the effects of the dynamic pressures on the lifting body that induce changes in their running attitudes and, particularly, to the interactions that arise with the water free surface. Such interactions on a side alter the pressure distribution on the body surface and, on the other side, produce waves that propagates in the far field downstream.

This hydrodynamic problem has been deeply studied both numerically and experimentally. Considering the latter approach the two components of the wave resistance, related to the breaking and the non- breaking waves, have been analyzed for a submerged hydrofoil [1, 2]. The pressure distribution at the wet surface of a planing plate has been measured for several configurations and conditions at the NASA research center [3]. Considering the numerical solution of the problem, a variety of methods have been proposed. Boundary Element Methods (BEM) relying on potential flow theory have been developed to solve related problems such as the performance of cavitating and supercavitating hydrofoils [4, 5, 6] or subcavitating hydrofoils interacting with a free surface [7] and are widely applied in design by optimization processes thanks to their inherent computational efficiency [8, 9]. Among potential flow based methods, there are also vortex based approaches relying on the so called Vortex Lattice Method (VLM), ini- 
tially developed to solve aerodynamics related problems [10]. Despite it is particularly suitable to predict lifting effects of a body, relatively few authors have developed VLM based approaches to predict the performance of lifting bodies such as planing hulls $[11,12,13,14]$. Other hydrodynamic solutions of planing hulls have been presented based on highfidelity Reynolds Averaged Navier Stokes (RANS) methods [15, 16, 17, 18, 19, 20, 21].

A VLM for the solution of lifting bodies, both submerged and surface piercing, is proposed. The mathematical formulation of the problem, i.e. the development of the suitable set of boundary conditions, is presented and the numerical scheme is described. An extensive validation of the developed VLM is then presented proving the accuracy of the solution. In particular the VLM is applied to the hydrodynamic analysis of submerged and surface piercing flat plates, both $2 \mathrm{D}$ and $3 \mathrm{D}$, to the solution of submerged, finite, NACA hydrofoils and to the performance prediction of a prismatic planing hull. The obtained results are compared with available experimental measurements and against results obtained from other computational methods.

\section{Free Surface Hydrodynamic Problem Formulation}

The fluid domain changes based on the position of the lifting body under analysis, according to the schematic representation shown in Fig. 11. Considering the fully submerged lifting body $S_{B}$, e.g. a hydrofoil, the fluid wake detached by its trailing edge $S_{W}$ needs to be included to fulfill the so-called Kutta condition and the free surface $S_{F} S$ is a continuous interface where stationary waves (both transverse and divergent) could develop. On the other hand, as the lifting body crosses the free surface, as e.g. in the case of a planing hull, a more complex wave pattern is generated since the fluid interface is forced to be attached to the bottom of the lifting body. Both cases have been developed for deep water condition, meaning that the sea bottom surface $S_{\infty}$ is far enough not be included into the model.

The solution is formulated in the framework of a socalled potential flow theory, i.e. assuming the flow to be ideal, irrotational and incompressible. Introducing the Cartesian reference system shown in Figure 2, consistent with the body, a velocity potential, scalar, function $\Phi$ can be defined so that $\nabla \Phi=\vec{v}$, being $\vec{v}=\left[U+v_{x}, v_{y}, v_{z}\right]$ the global flow velocity vector. In particular, such a potential function can be seen as the superimposition of a free stream potential $\phi_{0}=x \cdot U$ with a potential $\phi$ consequence of the disturbance to the flow generated by the presence of the body, resulting in $\Phi(x, y, z)=x \cdot U+\phi(x, y, z)$.
According to the mentioned hypothesis, the Laplace Eq. (11) hold in the fluid domain:

$$
\nabla^{2} \Phi=0, \quad \text { in } \mathfrak{D}
$$

A Neumann-type boundary condition on the body wet surface is imposed to ensure the non-penetration of the fluid particles. Being $\vec{n}=\left(n_{x}, n_{y}, n_{z}\right)$ the normal vector to the body surface, the normal velocity is null at the body boundaries according to Eq. (2):

$$
\nabla \Phi \cdot \vec{n}=\frac{\partial \phi}{\partial n}+U n_{x}=0, \text { at } S_{B}
$$

The perturbation needs to decay at a large distance $\vec{r}=\left(x_{r}, y_{r}, z_{r}\right)$ to the body. This radiation condition is expressed by Eq. 3:

$$
\lim _{\vec{r} \longrightarrow \infty} \nabla \Phi=0
$$

Considering the free surface as a function $z=$ $\eta(x, y)$, two boundary conditions hold, namely a kinematic and a dynamic condition. The first, according to Eq. 4, guarantees that the fluid particles belonging to the free surface will remain on that interface. The second is a direct consequence of the Bernoulli's theorem, written in Eq. 5, keeping constant the atmospheric pressure $p_{a t m}$ at the interface.

$$
\begin{gathered}
\frac{\partial \Phi}{\partial z}-\nabla \Phi \cdot \nabla \eta=0, \quad \text { on } z=\eta(x, y) \\
\frac{1}{2}(\nabla \Phi)^{2}-\frac{1}{2} U^{2}+g \eta=0, \quad \text { on } z=\eta(x, y)
\end{gathered}
$$

An additional boundary condition is required for a submerged lifting body, ensuring the uniqueness of the solution. From a physical perspective the Kutta condition imposes that the TE of the hydrofoil has to be a stagnation point, then $\Delta p_{T E}=0$. The free surface boundary conditions can be combined considering the gradient of the dynamic boundary condition, obtaining the following non-linear condition:

$$
\frac{1}{2} \nabla \Phi \cdot \nabla(\nabla \Phi)^{2}+g \frac{\partial \Phi}{\partial z}=0, \quad \text { on } z=\eta(x, y)
$$

Despite the advantage of being independent from the free surface elevation $\eta(x, y)$, which is part of the solution, Eq. (6) is still very complex to be fulfilled. For this reason, it can be further linearized by considering that it can be imposed at the undisturbed free surface level $z=0$, instead of the actual free surface $z=\operatorname{eta}(x, y)$ [22, 23], resulting in the following Eq. (7):

$$
U^{2} \frac{\partial \phi^{2}}{\partial x^{2}}+g \frac{\partial \phi}{\partial z}=0, \quad \text { on } z=0
$$




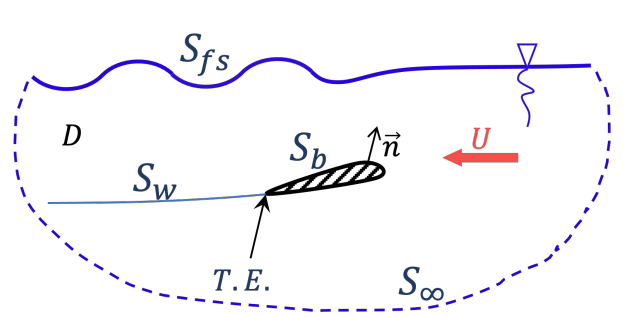

(a) $\frac{H_{S}}{b}=1.42 \%$

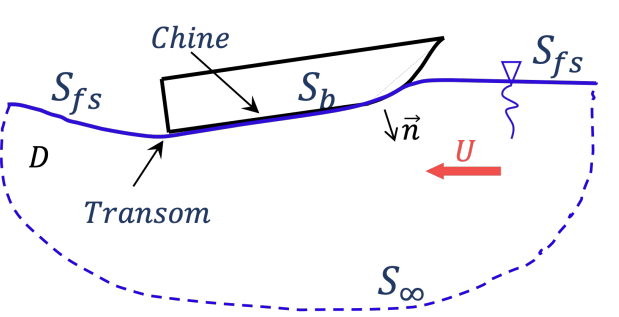

(b) $\frac{H_{S}}{b}=2.85 \%$

Figure 1: Schematic representation of the fluid domain for both the submerged (left) and surface-piercing (right) cases.

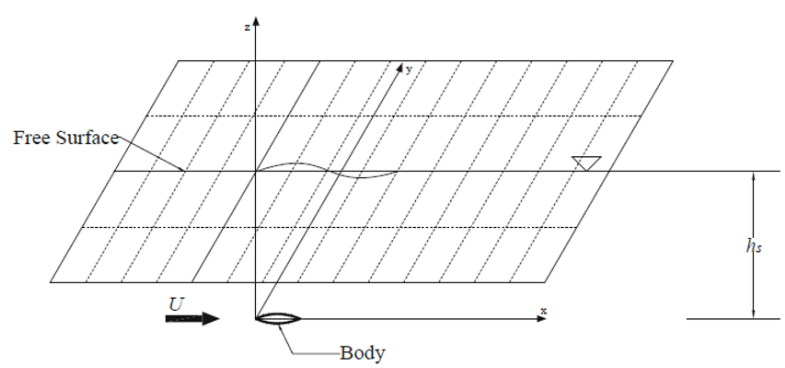

Figure 2: Reference system used for the formulation of the hydrodynamic problem.

According to the presented formulation, the Boundary Value Problem (BVP) for lifting bodies traveling close or across a free surface, in deep, water can be formulated in terms of two boundary conditions, namely Eq. (2) for the body and Eq. (7) for the linearized free surface.

\section{Solution trough the Vortex Lattice Method}

The potential function in a generic point of the considered domain can be written according to the Green equation, Eq. (8), based on the distribution of singular elements over the boundaries, as:

$$
\Phi(x, y, z)=\frac{1}{4 \pi} \int_{S} \mu \vec{n} \cdot \nabla\left(\frac{1}{r}\right) d S+U_{\infty} x
$$

being $S=S_{B}+\eta(x, y)$ and $\mu$ the strength of a constant distribution of dipoles. The numerical solution of the BVP is achieved by using a Vortex Lattice Method (VLM). According to this approach, each material surface (the body and the free surface) is discretized by using a lattice of vortex built by the socalled vortex rings, as shown in Fig. 3. Each of these vortex rings is made of four straight vortex filaments, each carrying a constant circulation $\Gamma_{i}$ strength, being circulation around the hydrofoil is defined as the

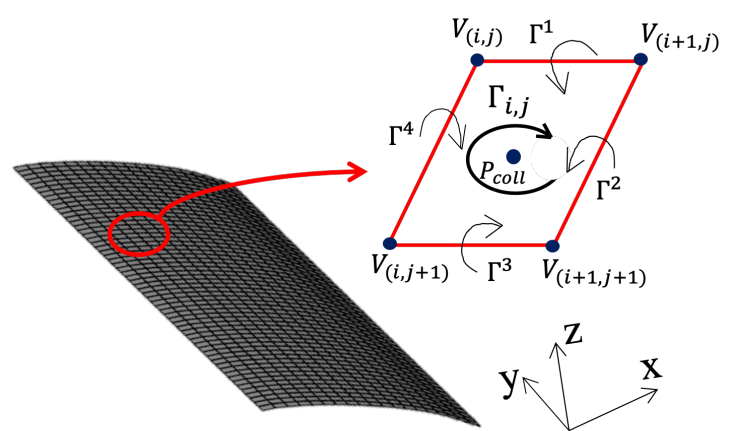

Figure 3: Generic representation of the VLM panel mesh discretization for a wing and particular of one of its vortex rings.

vorticity flux over a surface:

$$
\Gamma=\int_{S} \vec{\zeta} \cdot \vec{n} d S
$$

The vorticity is defined as the curl of the velocity $\vec{\zeta}=$ $\nabla \times \vec{v}$. A collocation point $P_{\text {coll }}$ is defined for each vortex ring as the center of the mean plane defined by the ring. The boundary conditions are enforced at those collocation points. The velocity induced by a vortex ring, that is equivalent to that induced by a constant distribution of dipoles, is found according to the Biot-Savart law, defined in Eq. (10) as:

$$
\vec{v}_{\text {ind }}=\frac{\Gamma}{4 \pi} \oint_{C} \frac{d \vec{l} \times \vec{r}}{r^{3}}
$$

being $\vec{l}$ and $\vec{r}$ the vector length of a vortex filament and the vector distance of the collocation point from the filament itself, respectively. Considering Eq. (10), the circulation strength $\Gamma$ of each vortex ring is the unknown to be found as solution of a linear system. The rest, called influence coefficient, only depends on the relative position of the collocation point 
with respect to the vortex ring and can be directly found.

Once the BVP has been solved, the total, linearized, pressure $p_{t}$ at a point $\mathrm{P}$, made of a static and a dynamic contribution, $p_{s}$ and $p_{d}$, respectively, can be found as:

$$
p_{t}=p_{s}+p_{d}=-\rho g\left(z_{p}+\eta\left(x_{p}, y_{p}\right)\right)-\rho U \frac{\partial \phi}{\partial x}
$$

The dynamic pressure in Eq. (11) is computed accounting for the tangential velocity jump $\Delta \vec{v}_{\text {tan }}$ at the lifting surface due to the presence of the vortex lattice. This contribution is computed considering the mean distribution of circulation $\vec{\gamma}_{i j}$ over a vortex ring of area $A_{i j}$ :

$$
\Delta \vec{v}_{t a n}=\vec{n}_{i j} \times \vec{\gamma}_{i j}=\frac{1}{A_{i j}} \sum_{k=1}^{4} \sigma \Gamma_{k} \cdot \vec{l}_{i j}
$$

being $\sigma=0.5$ everywhere but at the leading edge vortex filament for which $\sigma=1$. Then the total induced velocity at each collocation point is computed as:

$$
\vec{v}_{\text {ind }}=\vec{v}_{\text {ind }} \pm \frac{1}{2} \Delta \vec{v}_{\text {tan }}
$$

Considering a discretization made of $N \times M$ vortex rings, the lift and the induced drag are computed from the knowledge of the pressure as follows:

$$
\begin{gathered}
L=\sum_{i=1}^{N} \sum_{j=1}^{M} L_{i j}=\sum_{i=1}^{N} \sum_{j=1}^{M}-p_{T i j} \cdot A_{i j} \cdot n_{Z i j} \\
D=\sum_{i=1}^{N} \sum_{j=1}^{M} D_{i j}=\sum_{i=1}^{N} \sum_{j=1}^{M}-p_{T i j} \cdot A_{i j} \cdot n_{X i j}
\end{gathered}
$$

The Kutta condition, used in the case of a submerged hydrofoil, imposes that the strength of the vortexes at the TE is null, that is the circulation at the TE is released in the wake behind it. Such a further boundary condition is used to ensure the presence of a stagnation point at the TE (valid for attached flows without separation) and it is simply enforced by imposing $\Gamma_{T E}=\Gamma_{\text {Wake }}$.

As regards the free surface boundary condition, to prevent numerical damping of the free surface elevation, a four point derivation scheme is used for the term $\partial \phi / \partial x$ [22]. Moreover, the collocation points of the free surface are placed at a minimum distance $d h / c=0.1 \div 0.2$ with respect to the undisturbed free surface level $(\mathrm{z}=0)$ to let the generation of the complete wave pattern.

\section{Validation and verification of the VLM}

The method has been validate against available experimental data and other numerical results on several cases, namely a submerged flat plate, a submerged hydrofoil and a planing prismatic hull. In the following sections results of this validation process are presented and discussed, highlighting the accuracy of the obtained predictions.

\subsection{Submerged Finite Flat Plate}

The hydrodynamic performance of a deeply submerged flat plate, with $A R=3$ and $t / c=2.3 \%$, have been studied by using a CFD [24] at $R e=8 \cdot 10^{4}$. The comparison of the results in terms of lift and drag coefficients are displayed in Fig. 4 and Fig. 5, respectively. The obtained predictions have also been compared against theoretical results obtained by applying the $2 \mathrm{D}$ thin profile theory, corrected to include the three dimensional effects. In particular, the theoretical lift coefficient for an infinitely $\mathrm{AR}$ wing, i.e. a $2 \mathrm{D}$ case, is defined as:

$$
C_{L}^{2 D}=\frac{\partial C_{L}}{\partial \alpha} \cdot \sin (\alpha)=2 \pi \cdot \sin (\alpha)
$$

being $\alpha$ and $\partial C_{L} / \partial \alpha$ the angle of attack and the slope of the lift curve, respectively. Among the possible formulations, two have been selected for the AR correction of the lift curve slope, reported in Eq. (17) and Eq. (18), and one formula for the drag coefficient, reported in Eq. (19), respectively.

$$
\begin{gathered}
\frac{\partial C_{L}^{3 D}}{\partial \alpha}=f \cdot \frac{\frac{\partial C_{L}^{2 D}}{\partial \alpha} \frac{P}{l}}{1+\left(57.3 \cdot \frac{\frac{\partial C_{L}^{2 D}}{\partial \alpha}}{\pi A R \frac{P}{l}}\right)} \\
\frac{\partial C_{L}^{3 D}}{\partial \alpha}=\frac{\partial C_{L}^{2 D}}{\partial \alpha} \cdot \frac{A R}{A R+2} \\
C_{D}^{3 D}=\frac{2 C_{L}^{3 D}}{\pi e A R}
\end{gathered}
$$

In the previous equations, $P$ and $l$ are the half perimeter and the span of the wing, respectively, $f=$ $[0.98 ; 1]$ is a coefficient given as a function of the AR, and $e=1.78\left(1-0.045 A R^{0.68}\right)-0.64$ is the Oswald coefficient, which accounts for a correction for nonelliptic wings.

The lifting coefficient predicted by the proposed method is in very good agreement with the CFD results up to about $\alpha=12 \mathrm{deg}$, that is over all the linear trend of the curve. This is consistent with the assumptions of the potential flow theory used to develop the method. For higher angles of attack, predominant viscous effects rise, highlighting a larger separation 
among the viscous and inviscid predictions. Considering the drag coefficient, a relatively good agreement is found. Considering that only the induced drag is predicted by the VLM, a correction through Blausius formulation for the friction contribution $C_{f}$, valid for laminar flow regimes $\left(R e<2.5 \cdot 10^{5}\right)$, is applied. As for the lift coefficient, the drag coefficient predicted by the simplified formula is consistent with the other results only for small angles of attack, in the order of $\alpha<6$ deg.

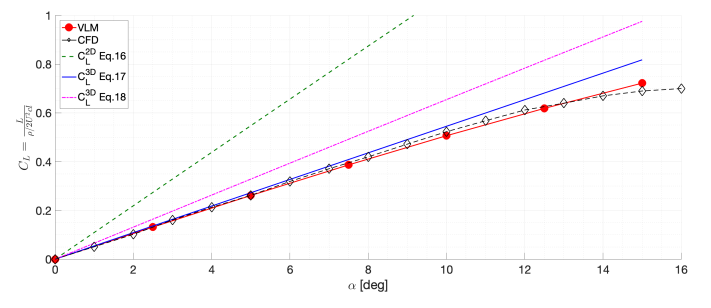

Figure 4: Comparison of the computed lift coefficients for the $A R=3$, deeply submerged, flat plate.

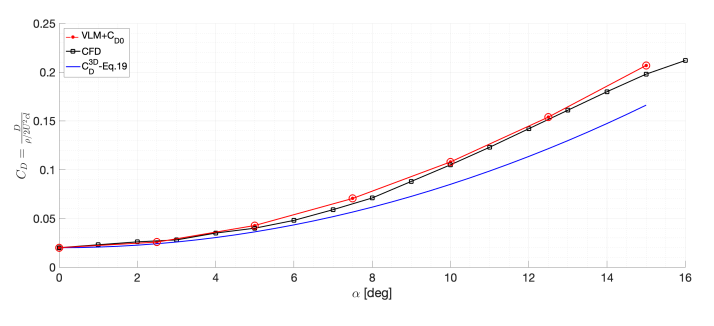

Figure 5: Comparison of the computed drag coefficients for the $\mathrm{AR}=3$, deeply submerged, flat plate.

\subsection{Submerged Infinite Flat Plate}

A 2D flat plate $(A R \rightarrow \infty)$ with $t / c=1 \%$ has been analyzed at $R e=10^{4}$ and results have been compared against available wind tunnel experiments [25], then neglecting the effect of the free surface. The comparisons of the lift coefficient is shown in Fig. 6. Again, the results are consistent within the linear trend of the lift curve. Being this case referred to a 2D flat plate, far from the free surface, the induced drag related to the three dimensional effect is null. The predicted pressure coefficient at the center line of the flat plate is compared against experimental measurements in Fig. 77. The pressure distribution is computed according to the velocity jump induced by the vortex distribution, as in Eq. (12). The relatively slight difference found on the face of the plate is ascribed to the effect of the thickness, that is not modeled in the present VLM (e.g. by using a source distribution superimposed to the vortex lattice).

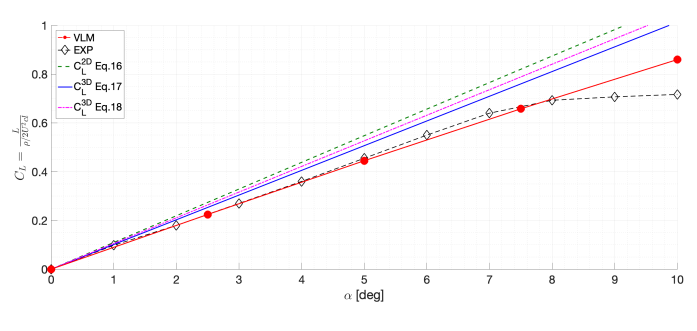

Figure 6: Comparison of the computed lift coefficients for the $A R \rightarrow \infty$, deeply submerged, flat plate.

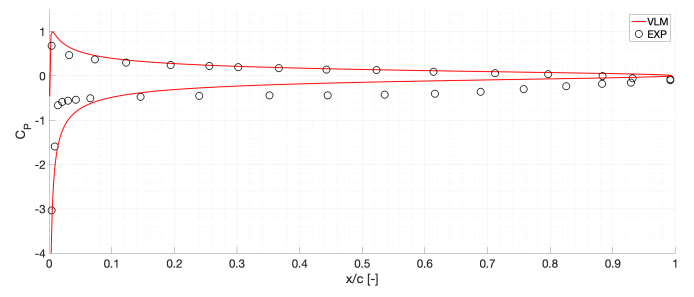

Figure 7: Comparison of the computed pressure distribution at the center line for the $A R \rightarrow \infty$, deeply submerged, flat plate.

\subsection{Submerged Finite NACA hydrofoil}

The predicted free surface elevation at the center line of a NACA profile, with a mean camber line $\mathrm{a}=0.8$, is compared against available numerical prediction [26] in Fig. 8. The hydrofoil, $A R=1.5$ and $t / c=0 \%$, is analyzed at a draught over chord ratio $T / c=1$, $\alpha=5 \mathrm{de} g$ and at a relatively high speed, corresponding to $F n=0.58$. The two methods, relying on the same theoretical approach, provide very similar results. The complete three dimensional, stationary, wave pattern generated by the hydrofoil is shown in Fig. 9. It is worth noticing that to avoid reflections of the waves a relatively large free surface domain has to be discretized. The dimensions of the free surface, especially its length behind the profile location, very much depends on the speed of the hydrofoil and on the submergence ratio since they affect the wave length and, consequently, the wave celerity. In general, it is in the order of several chord lengths $\left(L_{a f t} / c>10\right)$. The method has been used to analyze the effect of the angle of attack variation on the free surface elevation and on the overall performance of the hydrofoil. The predicted free surface profiles at the center line are shown in Fig. 10. As $\alpha$ increases a higher pressure disturbance is generated, resulting in a larger wave amplitude of the first wave hollow. Such a maximum, negative, wave amplitude is also anticipated due to the relative shift forward of the center of pressure of the hydrofoil. These characteristics are maintained over the whole development of the wave profile. Consis- 
tently, the lift and drag coefficients, referred in Fig. 11 to the 2D case, increase as $\alpha$ increases. While the $C_{L} / C_{L}^{2 D}$ ratio increment is in the extent of about $5 \%$ from $\alpha=0 \operatorname{deg}$ to $\alpha=10 \mathrm{deg}$, the $C_{D} / C_{D}^{2 D}$ ratio increases of about $14 \%$ over the same range of $\alpha$.

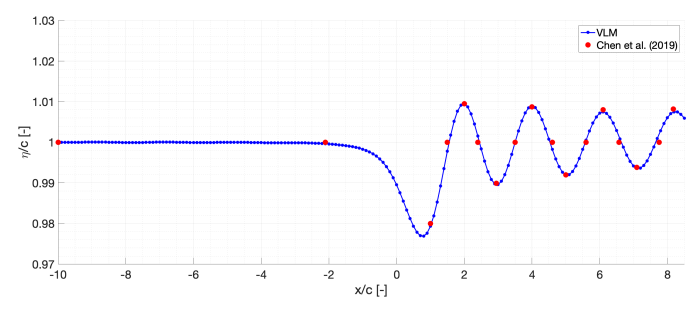

Figure 8: Wave profile at the symmetry plane of the free surface for the NACA $a=0.8$ mean camber line at $\mathrm{T} / \mathrm{c}=1$. Blue line with markers: VLM computation. Red circles: computation from [26]

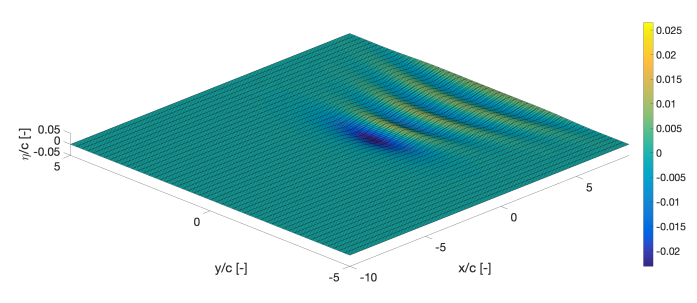

Figure 9: Global view of the computed free surface for the NACA $\mathrm{a}=0.8$ mean camber line at $\mathrm{T} / \mathrm{c}=1$.

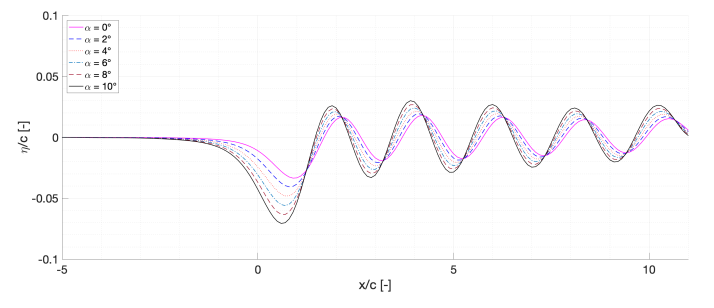

Figure 10: Wave profiles at the symmetry plane of the free surface for the NACA a $=0.8$ mean camber line at $\mathrm{T} / \mathrm{c}=1$ for several angle of attacks $\alpha$.

\subsection{Surface-Piercing Lifting Bodies}

The method has been conceived to account for surface piercing lifting bodies, as in the case of planing hulls. This is a very interesting application in the field of naval architecture since there are very few potential flow based methods developed to analyze it. The pressure coefficients at the half-span line of two planing flat plates experimentally tested at NASA [3] are

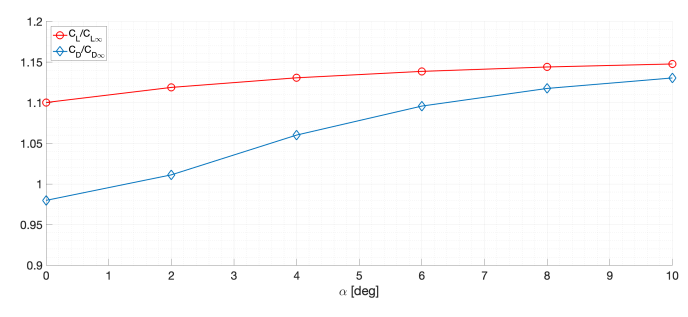

Figure 11: Variation of the $C_{L}$ and $C_{D}$ with respect to the $2 \mathrm{D}$ limit case for the NACA a $=0.8$ mean camber line for several angles of attack $\alpha$.

Table 1: Planing surfaces particulars and lift and drag coefficients comparison.

\begin{tabular}{l|r|r} 
& Case 1 & Case 2 \\
\hline $\mathrm{AR}$ & 2.87 & 1.07 \\
$F n_{B}$ & 10.11 & 12.14 \\
$\tau$ & 6.0 & 6.0 \\
$C_{L}^{V L M}$ & $6.10 \mathrm{E}-02$ & $8.80 \mathrm{E}-02$ \\
$C_{L}^{\text {Savitsky }}$ & $5.90 \mathrm{E}-02$ & $8.50 \mathrm{E}-02$ \\
$\epsilon\left(C_{L}\right)$ & $3.4 \%$ & $3.4 \%$ \\
$C_{D}^{V L M}$ & $6.40 \mathrm{E}-03$ & $9.30 \mathrm{E}-03$ \\
$C_{D}^{\text {Savitsky }}$ & $6.40 \mathrm{E}-03$ & $9.00 \mathrm{E}-03$ \\
$\epsilon\left(C_{D}\right)$ & $3.2 \%$ & $3.3 \%$ \\
\hline
\end{tabular}

compared to the numerical prediction carried out by using the proposed VLM and to those obtained by using another BEM [27], in Fig 12 and in Fig. 13, respectively. In Table 1, the main particulars of the two cases and the comparison of the corresponding lift and drag coefficients computed by the VLM and by the well known Savitsky's method [28] are reported. The Froude number is related to the beam (the span) of the surface, $F n_{B}=V / \sqrt{g B}$. The trim angle $\tau$ is the equivalent of the angle of attack $\alpha$ for an hydrofoil. The relative percentage errors of a quantity $\epsilon(x)$ are computed as:

$$
\epsilon(x)=100 \cdot \frac{\left|x_{r e f}-x\right|}{x_{r e f}}
$$

In both cases, the $C_{P}$ distributions are in very well agreement with the experimental measurements. In particular they better fit the experimental distribution especially close to the forward stagnation line, called spray root line, where the peak of the pressure occurs. This is a key feature for a reliable prediction of the overall planing hull performance. When compared to the Savitsky's predictions, used as term of reference, the percentage errors on both the lift and the drag coefficients are slightly beyond the $3 \%$, confirming that the global hydrodynamic performance are well predicted. 


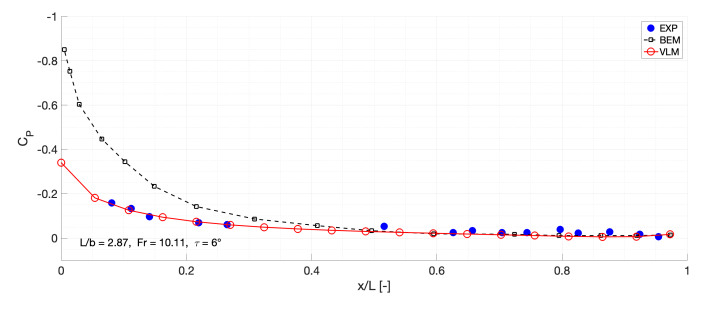

Figure 12: Pressure coefficient at the center line of a planing flat plate, $L / b=2.87, F r=10.11, \tau=$ 6deg. Blue circles: experimental measurements. Red solid line with circles: VLM computations. Black dashed line with squares: BEM computations from [27].

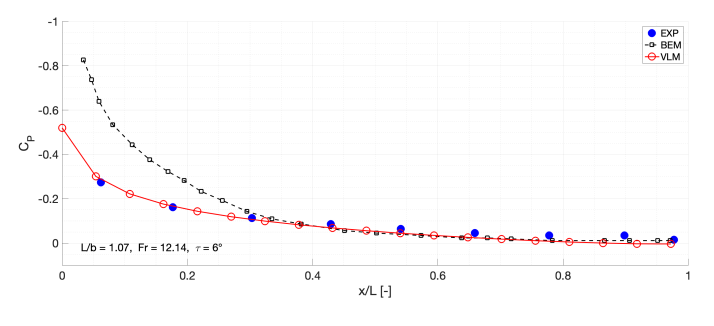

Figure 13: Pressure coefficient at the center line of a planing flat plate, $L / b=1.07, F r=12.14, \tau=$ $6 d e g$. Blue circles: experimental measurements. Red solid line with circles: VLM computations. Black dashed line with squares: BEM computations from [27].

The last analysis deals with prismatic planing hull. In this case a so called deadrise angle $\beta$ is introduced. This is similar to the dihedral angle typically used in wing design. The hull have been designed according to the Savitsky's method [28]. Its main particulars are listed in Table 2, being $L_{K}, L_{C}$ and $L_{M}$ the wet lengths at keel and chine and the mean wet length, respectively, $\mathrm{d}$ the draught at transom and $\lambda=\left(L_{K}+L_{C}\right) / 2 B$ the mean wet length ratio. The predicted lift and drag coefficients are compared to those obtained by Savitsky's method in Fig. 14 and in Fig. 15, respectively. The main differences between the two predictions are in the pre-planing regime, i.e. for $F n<1$. In this phase the hull is still supported by a relatively large hydrostatic force, as shown in Fig. ??. At higher speeds, $F n>1$, the dynamic pressure becomes prevalent compared to the hydrostatic component, supporting the hull that runs in fully planning regime. Since the VLM method has been developed particularly for this kind of flow regime, the obtained agreement is satisfactory. The steady wave patterns corresponding to the two Froude numbers $F n=[0.77,1.80]$ are shown in Fig. 16 and Fig.
Table 2: Planing surfaces particulars and lift and drag coefficients comparison.

\begin{tabular}{l|c|r} 
Particular & Units & Hull \\
\hline $\mathrm{L}$ & {$[\mathrm{m}]$} & 15 \\
$\mathrm{~B}$ & {$[\mathrm{~m}]$} & 4 \\
$\mathrm{~d}$ & {$[\mathrm{~m}]$} & 1 \\
$\tau$ & {$[\mathrm{deg}]$} & 5.0 \\
$\beta$ & {$[\mathrm{deg}]$} & 10.0 \\
$\lambda$ & {$[-]$} & 2.55 \\
$L_{K}$ & {$[\mathrm{~m}]$} & 11.47 \\
$L_{C}$ & {$[\mathrm{~m}]$} & 8.91 \\
$L_{M}$ & {$[\mathrm{~m}]$} & 10.2 \\
\hline
\end{tabular}

17. The free surface elevation is colored by using a color map from blue to yellow. Consistently with the known evidences on ship wave patter formation, in the pre-planing regime $(F n=0.77)$ there are both divergent and transverse waves (the latter being almost disappearing due to the relatively high speed). The so called Kelvin angle $\psi \approx 19 \mathrm{deg}$ from the bow apex, defining the existence domain of the generated waves in the plane of the free surface, is present. As the critical Froude number is overcome $F n>1$, the hull starts planing and the Kelvin sector narrows while the transverse waves completely disappear revealing a wave pattern made of divergent waves only.

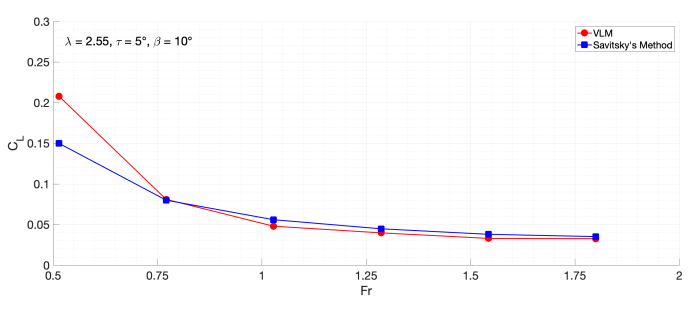

Figure 14: Lift coefficient vs Froude number for a prismatic planing hull, $\lambda=2.55, \tau=5 \mathrm{deg}, \beta=$ 10deg. Red: VLM computations. Blue: Savitsky's method.

\section{Conclusion}

The hydrodynamic problem of either submerged and surface-piercing lifting bodies has been solved in the framework of a potential flow theory. In particular, a Vortex Lattice Method has been developed to predict both the pressure distribution over these lifting surfaces and the steady free surface elevation generated by the presence of the body. The boundary value problem has been linearized in order to achieve a fast and reliable numerical solution. The method has been validated by comparison against available experimental data and against computational results 


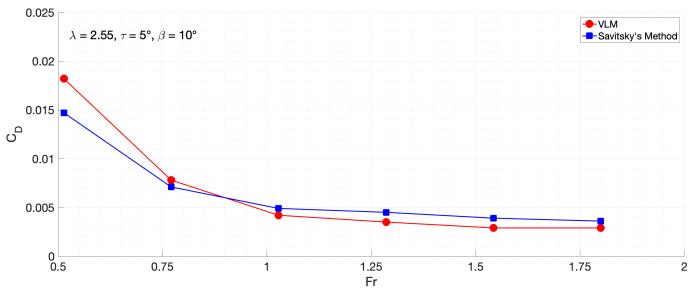

Figure 15: Drag coefficient vs Froude number for a prismatic planing hull, $\lambda=2.55, \tau=5 \mathrm{deg}, \beta=$ 10deg. Red: VLM computations. Blue: Savitsky's method.

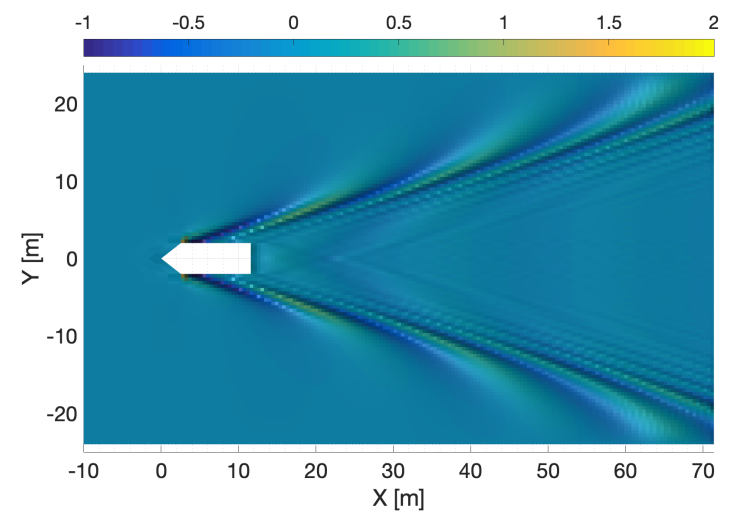

Figure 16: Complete wave pattern predicted for a prismatic planing hull, $\lambda=2.55, \tau=5 \mathrm{deg}, \beta=$ $10 \mathrm{deg}$, at $\mathrm{Fr}=0.77$. The wave elevation is displayed by using a colormap from blue $(\mathrm{z}=-1 \mathrm{~m})$ to yellow $(\mathrm{z}=2 \mathrm{~m})$.

from other sources, namely a conventional Boundary Element Method, a viscous RANS based solver, Savitsky's semi-empirical method for planing hulls and theoretical results from thin foil theory, including corrections for finite Aspect Ratios.

The validation process assesses the accuracy of the method, that results to be in good agreement with the majority of the analyzed cases in terms of pressure, lift and drag coefficients. The method has been further applied to analyzed the free surface formation of both a submerged hydrofoil, i.e. a NACA a $=0.8$ mean camber line, and a prismatic planing hull. The obtained trends of the free surface, despite not directly validated against experimental measurements, are consistent with the theoretical knowledge on these phenomena, then confirming the VLM predictions.

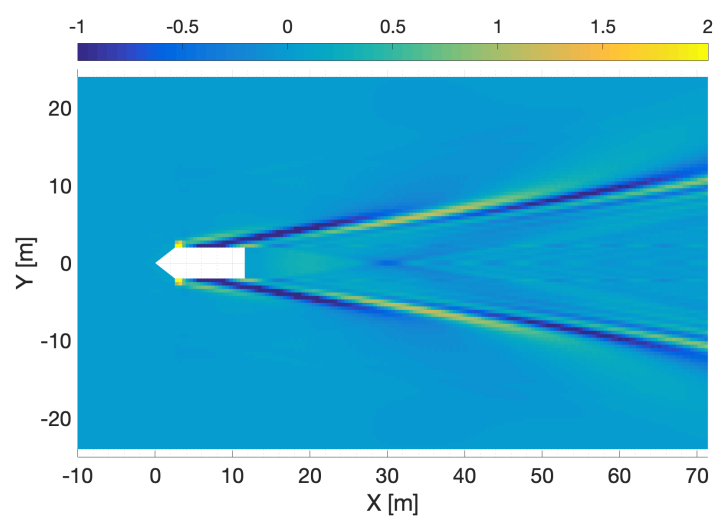

Figure 17: Complete wave pattern predicted for a prismatic planing hull, $\lambda=2.55, \tau=5 \mathrm{deg}, \beta=$ $10 \mathrm{deg}$, at $\mathrm{Fr}=1.80$. The wave elevation is displayed by using a colormap from blue $(\mathrm{z}=-1 \mathrm{~m})$ to yellow $(\mathrm{z}=2 \mathrm{~m})$.

\section{References}

[1] JH Duncan. An experimental investigation of breaking waves produced by a towed hydrofoil. Proceedings of the Royal Society of London. A. Mathematical and Physical Sciences, 377(1770):331--348, 1981.

[2] James H Duncan. The breaking and nonbreaking wave resistance of a two-dimensional hydrofoil. Journal of fluid mechanics, 126:507$-520,1983$.

[3] Walter J Kapryan and George M Boyd Jr. Hydrodynamic pressure distributions obtained during a planing investigation of five related prismatic surfaces. Technical report.

[4] Spyros A Kinnas and Neal E Fine. A numerical nonlinear analysis of the flow around twoand three-dimensional partially cavitating hydrofoils. Journal of Fluid Mechanics, 254:151$-181,1993$.

[5] S Bal and SA Kinnas. A bem for the prediction of free surface effects on cavitating hydrofoils. Computational Mechanics, 28(3):260-274, 2002.

[6] Giuliano Vernengo, Luca Bonfiglio, Stefano Gaggero, and Stefano Brizzolara. Physics-based design by optimization of unconventional supercavitating hydrofoils. Journal of Ship Research, 60(04):187--202, 2016.

[7] Carl-Erik Janson. Linear and non-linear potential-flow calculations of free-surface 
waves with lift and induced drag. Proceedings of the Institution of Mechanical Engineers, Part C: Journal of Mechanical Engineering Science, 214(6):801--812, 2000.

[8] Konstantinos V Kostas, Alexandros I Ginnis, Constantinos G Politis, and Panagiotis D Kaklis. Shape-optimization of $2 \mathrm{~d}$ hydrofoils using an isogeometric bem solver. Computer-Aided Design, 82:79--87, 2017.

[9] JO Royset, L Bonfiglio, G Vernengo, and S Brizzolara. Risk-adaptive set-based design and applications to shaping a hydrofoil. Journal of Mechanical Design, 139(10):101403, 2017.

[10] Joseph Katz and Allen Plotkin. Low-speed aerodynamics, volume 13. Cambridge university press, 2001.

[11] Canhai Lai and Armin W Troesch. Modeling issues related to the hydrodynamics of threedimensional steady planing. Journal of Ship research, 39(01):1--24, 1995.

[12] Brant R Savander, Stephen M Scorpio, and Robert K Taylor. Steady hydrodynamic analysis of planing surfaces. Journal of ship research, 46(04):248--279, 2002.

[13] Gunther Migeotte. Design and optimization of hydrofoil-assisted catamarans. $\mathrm{PhD}$ thesis, Stellenbosch: Stellenbosch University, 2002.

[14] Stefano Brizzolara and Giuliano Vernengo. A three-dimensional vortex method for the hydrodynamic solution of planing cambered dihedral surfaces. Engineering Analysis with Boundary Elements, 63:15--29, 2016.

[15] D Villa, S Gaggero, and M Ferrando. An open source approach for the prediction of planing hull resistance. In Proceedings of the 10th symposium on high speed machine vehicles, Naples, Italy, pages 15--17, 2014.

[16] Fabio De Luca, Simone Mancini, Salvatore Miranda, and Claudio Pensa. An extended verification and validation study of cfd simulations for planing hulls. Journal of Ship Research, 60(02):101--118, 2016.

[17] Agostino De Marco, Simone Mancini, Salvatore Miranda, Raffaele Scognamiglio, and Luigi Vitiello. Experimental and numerical hydrodynamic analysis of a stepped planing hull. $A p$ plied Ocean Research, 64:135--154, 2017.
[18] Jiahui Li, Luca Bonfiglio, and Stefano Brizzolara. Verification and validation study of openfoam on the generic prismatic planing hull form. In MARINE VIII: proceedings of the VIII International Conference on Computational Methods in Marine Engineering, pages 428--440. CIMNE, 2019.

[19] Rasul Niazmand Bilandi, Abbas Dashtimanesh, and Sasan Tavakoli. Hydrodynamic study of heeled double-stepped planing hulls using cfd and $2 \mathrm{~d}+\mathrm{t}$ method. Ocean Engineering, 196:106813, 2020.

[20] Carolyn Judge, Maysam Mousaviraad, Frederick Stern, Evan Lee, Anne Fullerton, Jayson Geiser, Christine Schleicher, Craig Merrill, Charles Weil, Jason Morin, et al. Experiments and cfd of a high-speed deep-v planing hull---part i: Calm water. Applied Ocean Research, 96:102060, 2020.

[21] Azim Hosseini, Sasan Tavakoli, Abbas Dashtimanesh, Prasanta K Sahoo, and Mihkel Kõrgesaar. Performance prediction of a hard-chine planing hull by employing different cfd models. Journal of Marine Science and Engineering, 9(5):481, 2021.

[22] CW Dawson. A practical computer method for solving ship-wave problems. In Proceedings of Second International Conference on Numerical Ship Hydrodynamics, pages 30--38, 1977.

[23] GD Thiart. Vortex lattice method for a straight hydrofoil near a free surface. International shipbuilding progress, 44(437):5--26, 1997.

[24] Khurshid Malik, Waqar Asrar, and Erwin Sulaeman. Low reynolds number numerical simulation of the aerodynamic coefficients of a $3 \mathrm{~d}$ wing. International Journal of Aviation, Aeronautics, and Aerospace, 5(1):8, 2018.

[25] Justin Winslow, Hikaru Otsuka, Bharath Govindarajan, and Inderjit Chopra. Basic understanding of airfoil characteristics at low reynolds numbers (10 4--10 5). Journal of Aircraft, 55(3):1050--1061, 2018.

[26] Qiang Chen, Chen-Jun Yang, and Xiao-Qian Dong. A vortex-lattice modeling approach for free-surface effects on submerged bodies and propellers. In Practical Design of Ships and Other Floating Structures, pages 636--652. Springer, 2019. 
[27] Ahmad Reza Kohansal and Hassan Ghassemi. A numerical modeling of hydrodynamic characteristics of various planing hull forms. Ocean Engineering, 37(5-6):498--510, 2010.

[28] Daniel Savitsky. Hydrodynamic design of planing hulls. Marine Technology and SNAME News, 1(04):71--95, 1964.

\section{Contribution of individual authors to the creation of a scientific article (ghostwriting policy)}

Raffaele Solari: Methodology, software, investigation, validation, writing - review and editing.

Patrizia Bagnerini: Methodology, supervision, writ- ing - review and editing.

Giuliano Vernengo: Conceptualization, methodology, visualization, supervision, writing - original draft, review and editing.

\section{Creative Commons Attribution License 4.0 (Attribution 4.0 International, CC BY 4.0)}

This article is published under the terms of the Creative Commons Attribution License 4.0

https://creativecommons.org/licenses/by/4.0/deed.en US 